\title{
Finite element analysis of normal pressure hydrocephalus: influence of CSF content and anisotropy in permeability
}

\author{
K. Shahima*, J.-M. Drezet ${ }^{\mathrm{a}}$, J.-F. Molinari ${ }^{\mathrm{b}}$, R. Sinkus ${ }^{\mathrm{c}}$ and S. Momjian ${ }^{\mathrm{d}}$ \\ ${ }^{a}$ LSMX, Ecole Polytechnique Fédérale de Lausanne, Lausanne, Switzerland; ${ }^{b}$ LSMS, Ecole Polytechnique Fédérale de Lausanne, \\ Lausanne, Switzerland; ${ }^{c}$ Laboratoire Ondes et Acoustique, ESPCI, Paris, France; ${ }^{d}$ University Hospitals of Geneva and University of \\ Geneva, Switzerland
}

(Received 23 December 2009; final version received 27 May 2010)

\begin{abstract}
Hydrocephalus is a cerebral disease where brain ventricles enlarge and compress the brain parenchyma towards the skull leading to symptoms like dementia, walking disorder and incontinence. The origin of normal pressure hydrocephalus is still obscure. In order to study this disease, a finite element model is built using the geometries of the ventricles and the skull measured by magnetic resonance imaging. The brain parenchyma is modelled as a porous medium fully saturated with cerebrospinal fluid (CSF) using Biot's theory of consolidation (1941). Owing to the existence of bundles of axons, the brain parenchyma shows locally anisotropic behaviour. Indeed, permeability is higher along the fibre tracts in the white matter region. In contrast, grey matter is isotropic. Diffusion tensor imaging is used to establish the local CSF content and the fibre tracts direction together with the associated local frame where the permeability coefficients are given by dedicated formulas. The present study shows that both inhomogeneous CSF content and anisotropy in permeability have a great influence on the CSF flow pattern through the parenchyma under an imposed pressure gradient between the ventricles and the subarachnoid spaces.
\end{abstract}

Keywords: biomechanics; hydrocephalus; brain parenchyma; permeability; anisotropy; finite element modelling

\section{Introduction}

Hydrocephalus is a cerebral disease wherein the brain ventricles dilate and compress the parenchyma. In noncommunicating or obstructive hydrocephalus, there is a blockage of the cerebrospinal fluid (CSF) circulation inside the ventricular system, usually in the aqueduct of Sylvius. In communicating hydrocephalus, the obstruction to the CSF flow occurs not only inside the ventricular system (Hakim 1971; Peña 2002) but also in the arachnoid granulations where CSF is absorbed by the venous system. In these two forms of hydrocephalus, a raised intracranial pressure (ICP) is observed together with a low-pressure gradient. Normal pressure hydrocephalus (NPH) is a chronic form of hydrocephalus where the ventricles enlarge although the CSF pressure remains close to normal $(\sim 10 \mathrm{mmHg})$ within the ventricular system and the brain (Hakim and Adams 1965; Levine 2008). Although the presence of a pressure gradient across the cerebral mantle is expected in obstructive hydrocephalus, such a gradient has been reported to be small ( $\sim 1 \mathrm{mmHg}$ or lower) (Levine 2008). This gradient would be even smaller in communicating hydrocephalus and particularly in NPH, which renders the mechanism of ventricular dilatation in the latter condition notoriously mysterious. Figure 1 shows the ventricle dilation on an axial section in a NPH patient showing clinical signs of urinary incontinence and dementia compared to the normal brain captured by computed tomography (CT). The volume of the ventricles is almost double.

In the present study, we focus on NPH as its mechanisms are yet unclear compared to the obstructive and communicating cases which are caused by CSF flow obstructions. The pathological manifestations of NPH are gradual memory loss (dementia), balance disorder (ataxia), urinary incontinence and general slowing of activity (Hakim and Adams 1965). The first 'mechanical' explanation of NPH was given by Hakim and Adams (1965), who asserted that a given pressure exerts a greater force in a large ventricular system than in a smaller one. In his recent explanation of the NPH mechanisms, Levine (2008) pointed out that a CSF pressure mini-gradient exists within the cerebral mantle in order to balance the CSF absorption by the blood vessels. He introduced a new index called degree of pressure transmission. With a high degree of transmission or higher incompressibility of brain parenchyma, acute hydrocephalus with high ICP is likely to occur, while higher compressibility of the brain parenchyma will result in NPH.

In the present study, a model of the brain is built in a finite element (FE) program. Magnetic Resonance

*Corresponding author. Email: kamal.shahim@epfl.ch 
(a)

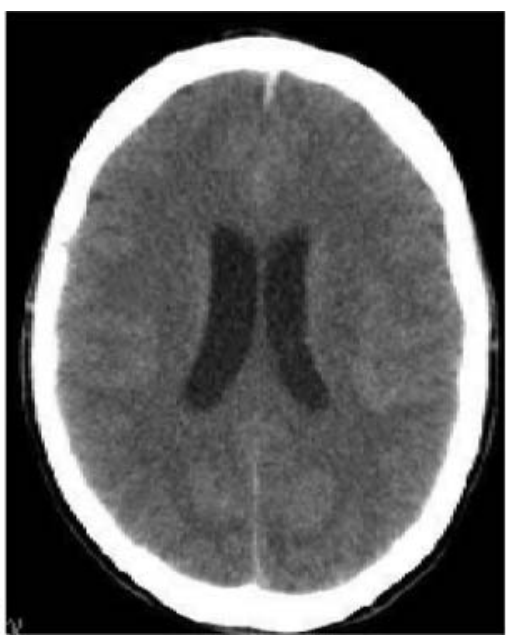

(b)

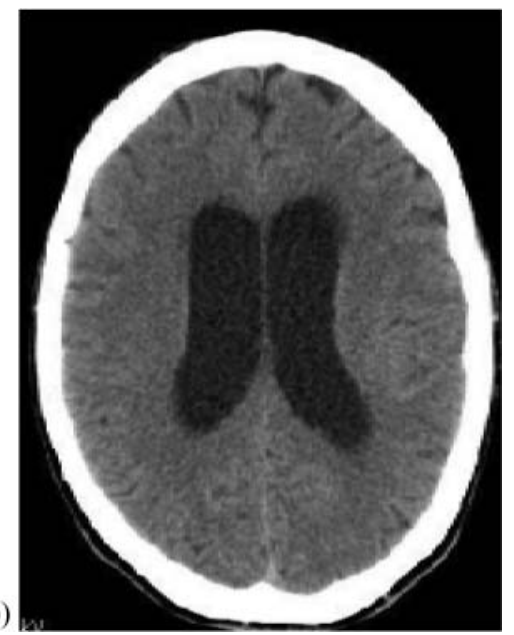

Figure 1. Computed tomography (CT) scans (axial sections) of normal (a) and dilated (b) ventricles in NPH (Momjian and Bichsel 2008).

Imaging (MRI) and Diffusion Tensor Imaging (DTI) data are used to determine the computation domain and the CSF content together with the permeability. MRI is used to determine the geometry of the brain parenchyma between the skull and the ventricles. With the use of different software (Matlab ${ }^{\mathrm{TM}}(\mathrm{http} / / /$ www.Matlab.com) and MRIcro $^{\mathrm{TM}}$ (http://www.MRIcro.com)) it is possible to build 3D geometries of the brain parenchyma from MRI images provided a good contrast between the ventricles and the subarachnoid spaces (SAS) is obtained. For the sake of simplicity and short computation times, one axial section of the brain is considered in the present study.

DTI is a non-invasive technique that measures water diffusion rates and thus gives an indication of the underlying tissue microstructure. Basser et al. (1994) proposed to represent the water diffusion in all the directions of space as a tensor in each voxel. The underlying assumption of diffusion-based tractography is that the water diffusion coefficient is different, higher in the present case, along the axis of the axon bundles rather than perpendicular to it. This aspect is used to construct the 3D pattern of the axon tracts using the so-called tractography technique (Melhem et al. 2002).

The brain parenchyma represents per se a heterogeneous medium and the presence of bundles of axons, i.e. fibre tracts, in the white matter gives birth to anisotropy not only in permeability but also in the elastic properties (Larrat et al. 2007). Both properties exhibit the so-called transverse isotropic (TI) behaviour as, for instance, in bone or muscle. In white matter, the elasticity and permeability properties are different along the fibre tracts direction than perpendicular to them as illustrated in Figure 2(a). The local frame $(1,2,3)$ is aligned with the direction of neuron fibres whereas $(x, y, z)$ is the reference frame. The plane perpendicular to the fibre tracts, plane (1 and 2), is assumed to exhibit isotropic elasticity and permeability, as represented schematically in Figure 2. The difficulty resides in the determination of the permeability coefficients in each direction.

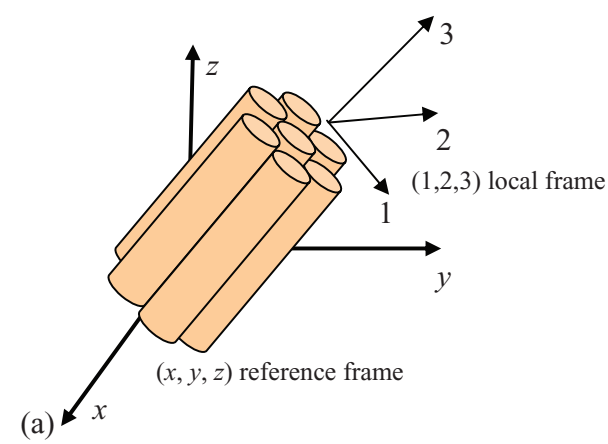

(b)

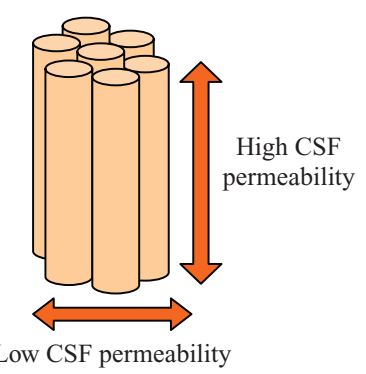

Figure 2. Schematic representation of the fibre tracts in white matter and the associated non-isotropic properties (Larrat et al. 2007). 
There have been many attempts to model the brain analytically and numerically using the FE technique. Different kinds of constitutive equations have been considered (Peña et al. 1999; Tenti et al. 1999; Peña 2002; Sivaloganathan et al. 2005b; Wirth 2005; Dutta-Roy et al. 2008). The model by Wirth (2005) incorporates strain dependence of the brain's permeability in three different geometries. In her work, the skull and ventricles have been simplified to spherical and cylindrical geometries. Peña et al. $(1999,2002)$ enlightened the CSF accumulation around the ventricular horns with the help of an FE model under an applied pressure gradient. By introducing large deformation (LD) theory and a hyperelastic constitutive model in a 3D model of the brain, Dutta-Roy et al. (2008) tested different mechanical approaches such as compressible solid parts, single phase or biphasic models to understand NPH, but the brain parenchyma was modelled as isotropic. With the help of a 2D isotropic model, Peña et al. (1999) and Momjian and Bichsel (2006) showed that the greatest stress concentrations and the largest deformations occur at the anterolateral angle of the frontal horn.

From the mechanical point of view, elastic and poroelastic (Biot's theory of consolidation (1941)) have been used assuming isotropic and homogenous elasticity and permeability tensors (Nagashima et al. 1987; Kaczmarek et al. 1997; Smillie et al. 2005; Momjian and Bichsel 2006). Some non-linearity has later been introduced through either a dilation-dependent Young's modulus (Dutta-Roy et al. 2008; Momjian and Bichsel 2008) or a dilationdependent permeability (Kaczmarek et al. 1997; Sivaloganathan et al. 2005a) of the brain parenchyma. So far, the brain parenchyma has always been considered as a porous medium with isotropic and homogenous permeability. To our knowledge, the influence of the non-uniform CSF content and anisotropy in permeability has never been studied. The present work combines the results of two non-invasive imaging techniques, MRI and DTI, together with an FE model, the goal being to evaluate the importance of the non-uniform CSF content and non-isotropic permeability in the case of NPH. To do so, the response of the cerebral mantle to an imposed CSF pressure gradient of $5 \mathrm{mmHg}$ is studied.

The DTI data are presented first, together with the link with permeability. Then the FE model using those data is presented together with the constitutive equations and the applied boundary conditions. Two different numerical cases are also defined in this section. Results and discussion are then presented and compared in the last section.

\section{Experimental data}

\subsection{Diffusion tensor imaging (DTI)}

An SE-EPI (Echo planar) sequence (field of view, $200 \mathrm{~mm}$; echo time, $65 \mathrm{~ms}$; b-factor, $1000 \mathrm{~s} / \mathrm{mm}^{2}$; six non-collinear directions; 27 contiguous, 5-mm slices) was used. The 128 matrix is the standard matrix for the SE-EPI sequence and offers a good signal to noise ratio (SNR). The acquisition was repeated twice to improve the SNR. The total time for DTI acquisition was about $3 \mathrm{~min}$. Geometric distortions as a result of eddy currents were first corrected. Diffusion was measured in terms of the apparent-diffusion coefficient and estimated for each voxel with non-linear regression (Basser and Pierpaoli 1998). For each estimate, the three orthogonal eigenvectors and their related positive eigenvalues were calculated. The principal direction of diffusion is given by the eigenvector that corresponds to the largest eigenvalue.

As diffusion is considered to be anisotropic, it is no longer characterised by a single scalar coefficient, but requires a $3 \times 3$ positive symmetric matrix, $D$, i.e. six scalars. When this tensor is expressed in the fibre tracts direction local frame $(1,2,3)$, it becomes diagonal with three eigenvalues $\left(D_{1}, D_{2}, D_{3}\right)$. The mean diffusivity (MD) $\left(\mathrm{m}^{2} / \mathrm{s}\right)$ and the fractional anisotropy in diffusion $\left(\mathrm{F}_{\mathrm{AD}}\right)$ are defined respectively as follows (Bihan et al. 1993):

$$
\begin{aligned}
\mathrm{MD}= & \bar{D}=\frac{D_{1}+D_{2}+D_{3}}{3} \\
\mathrm{~F}_{\mathrm{AD}}= & \sqrt{\frac{3}{2}} \sqrt{\frac{\left(D_{1}-\bar{D}\right)^{2}+\left(D_{2}-\bar{D}\right)^{2}+\left(D_{3}-\bar{D}\right)^{2}}{D_{1}^{2}+D_{2}^{2}+D_{3}^{2}}} \in \\
& {[0,1] . }
\end{aligned}
$$

Figure 3 shows these two quantities over the axial slice of the DTI data set of a human brain measured at Hôpitaux Universitaires de Genève (HUG) and used for the present study. MD allows distinguishing between cerebrospinal fluid content, where MD is high, and brain tissue, where MD is low. On the other hand, high fractional anisotropy $\left(\mathrm{F}_{\mathrm{AD}}\right)$ indicates the presence of white matter, because the directionality of the axon bundles allows for a faster diffusion along the neurons than across them (cf. Figure 2). Higher $\mathrm{F}_{\mathrm{AD}}$ values indicate increased anisotropic diffusion, as can be seen inside the corpus callosum where $\mathrm{F}_{\mathrm{AD}}$ is close to 1.0 (Melhem et al. 2002). Surrounding the ventricles, corpus callosum and corona radiata present the highest values of $\mathrm{F}_{\mathrm{AD}}$. On the other hand, $\mathrm{F}_{\mathrm{AD}}$ is close to zero in gray matter.

To distinguish between white and gray matters inside the parenchyma, a threshold based on the level of anisotropy is used similarly to Green et al. (2008). Voxels having $\mathrm{F}_{\mathrm{AD}}$ value above 0.25 are considered as white matter, whereas voxels with $\mathrm{F}_{\mathrm{AD}}$ value below 0.25 are considered as gray matter. This value of 0.25 comes from the uncertainties associated with the DTI measurements.

\subsection{CSF content}

As mean diffusivity highlights the CSF content, a fit is done to relate these two variables. The range of MD is from 
(a)

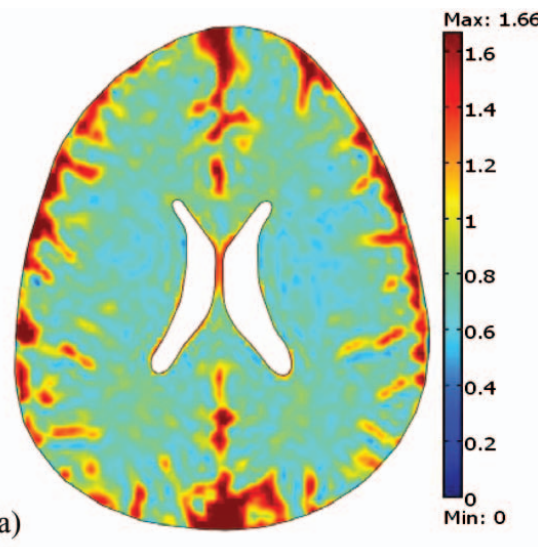

(b)

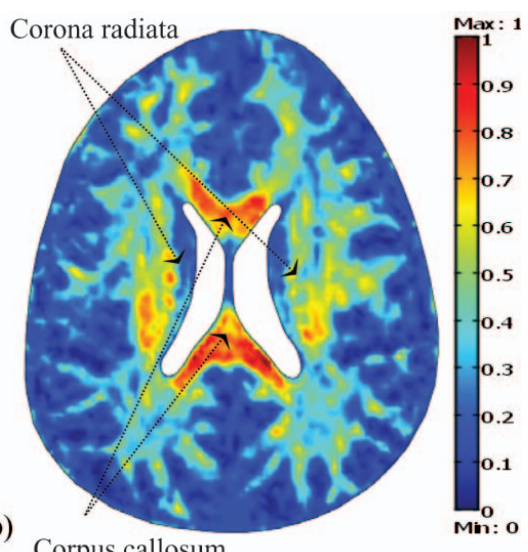

Figure 3. (a) Mean diffusivity (MD) $\left(10^{-3} \mathrm{~mm}^{2} / \mathrm{s}\right)$, and (b) fractional anisotropy in diffusion $\left(\mathrm{F}_{\mathrm{AD}}\right)$.

0 to $1.667 \times 10^{-3} \mathrm{~mm}^{2} / \mathrm{s}$, while the CSF content varies from 0 to $100 \%$. In addition, the average CSF content in the brain parenchyma is taken as $18 \%$ and is assigned to the mean value of MD, $0.806 \times 10^{-3} \mathrm{~mm}^{2} / \mathrm{s}$, over the slice. The polynomial fit presented in Figure 4 is used to link $f_{0}$ (in percentage) with MD expressed in units of $10^{-3} \mathrm{~mm}^{2} / \mathrm{s}$ :

$$
f_{0}=9.633(\mathrm{MD})^{3}+19.94(\mathrm{MD})^{2} .
$$

\subsection{Diffusion and permeability}

The link between diffusion and permeability in the brain is not well established. Similar to what Gupta et al. (2009) did for the permeability of SAS, we use the Westhuizen and Du Plessis (1994) formulas for the parallel and perpendicular permeability coefficients in the brain parenchyma:

$$
k_{\text {para }}=\frac{f^{2}(\pi+2.157(1-f))}{48(1-f)^{2}} d_{w}^{2},\left[m^{2}\right] \text {, }
$$

$$
k_{\text {perp }}=\frac{\pi f(1-\sqrt{1-f})^{2}}{24(1-f)^{3 / 2}} d_{w}^{2},\left[m^{2}\right],
$$

where $f$ and $d_{\mathrm{w}}$ are the CSF content and diameter of axon fibre tracts, respectively. White matter permeability is assumed to be TI and the direction of highest permeability is similar to the direction of highest diffusion. For gray matter, the isotropic Kozeny-Carman permeability (Carman 1937) is used:

$$
k_{\text {gray }}=\frac{f^{3}}{180(1-f)^{2}} d_{g}^{2},\left[m^{2}\right] \text {, }
$$

where $d_{g}$ represents the distance between gray cell bodies. In white matter the fibre tract diameter falls within the range of 1 to $10 \mu \mathrm{m}$ (Aboitiz et al. 1992; Yaniv et al. 2008). The average $5 \mu \mathrm{m}$ is used in Equations (4-5). For gray matter, the related dimension accounts for the passage size between gray cells and is also of the order of 5 $\mu \mathrm{m}$. The three permeability coefficients $k_{\text {para }}, k_{\text {perp }}$ and $k_{\text {gray }}$ are plotted in Figure 5 as a function of the CSF content

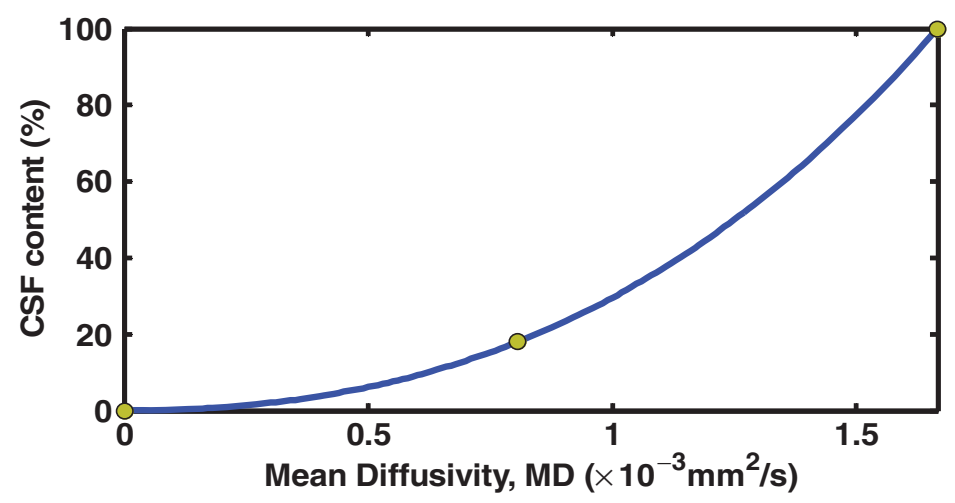

Figure 4. CSF content (\%) versus mean diffusivity (MD). 


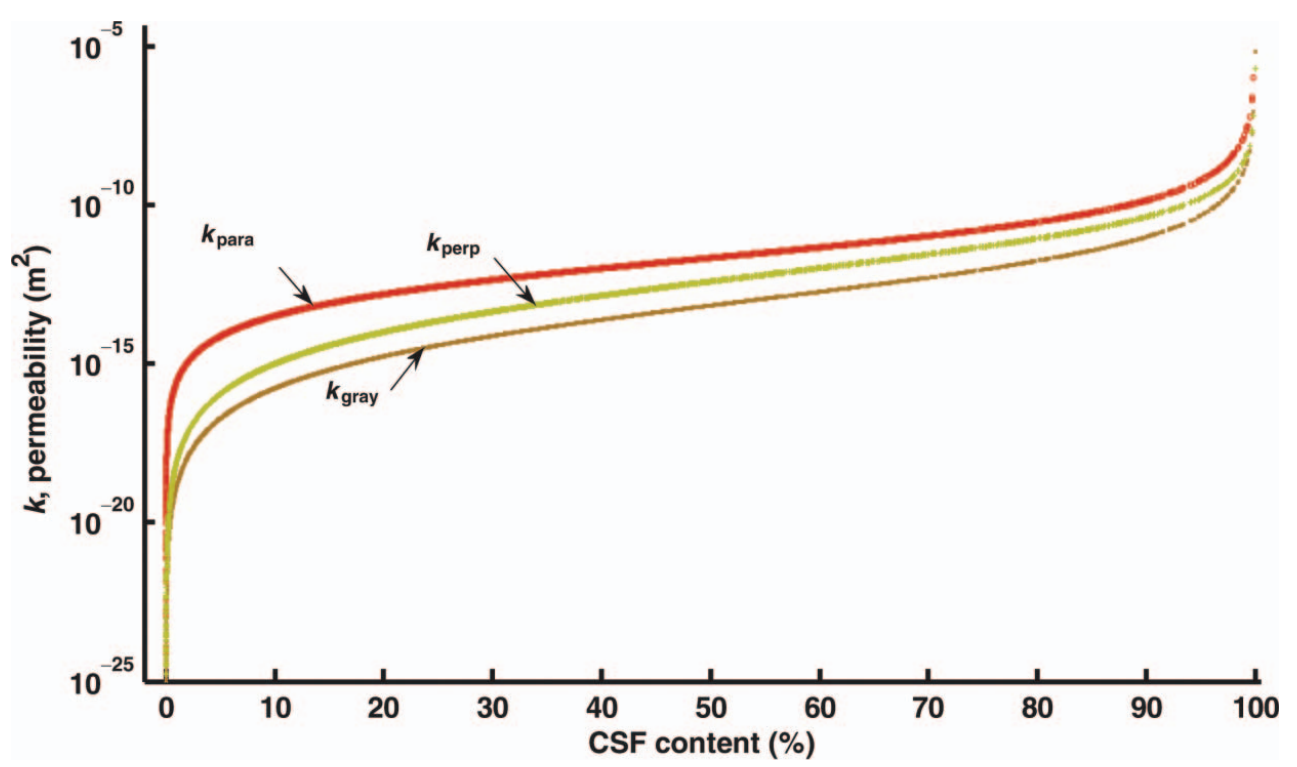

Figure 5. Permeability coefficients for gray and white matters versus CSF content (\%).

using a log scale as they vary over orders of magnitude. All coefficients tend to be infinite when CSF content tends to $100 \%$ and to 0 when CSF content tends to 0 . In addition, $k_{\text {para }}$ is always larger than $k_{\text {perp }}$, which is larger than $k_{\text {gray }}$. The degree of anisotropy is very large for low CSF content ( 2 to $30 \%$ ) and decreases when CSF content increases. The average CSF content inside the parenchyma is reported in the literature as $18 \%$. This yields $k_{\text {para }}=$ $10^{-13} \mathrm{~m}^{2}$ and $k_{\text {gray }}=10^{-15} \mathrm{~m}^{2}$. Basser used permeability values of $7.510^{-15} \mathrm{~m}^{2}$ for white matter and $5.010^{-15}$ $\mathrm{m}^{2}$ for gray matter (Basser 1992; Kaczmarek et al. 1997; Linninger et al. 2009) and Kaczmarek considered 1.6 10 $10^{-14}$ $\mathrm{m}^{2}$ and $1.610^{-16} \mathrm{~m}^{2}$, respectively, with a ratio of 100 between them (Basser 1992; Kaczmarek et al. 1997; Linninger et al. 2009). Linninger assumed an isotropic permeability of $1.010^{-14} \mathrm{~m}^{2}$ in the parenchyma (Basser 1992; Kaczmarek et al. 1997; Linninger et al. 2009). As a conclusion, the permeability coefficients given by Equations (4) to (6) correlate well with the values found in the literature.

Using the permeability coefficients defined in Equations (4) to (6) and the relationship between MD and CSF content (Equation (3)), a simple but realistic link is established between the DTI data and both the CSF content and the permeability coefficients.

\section{Finite element modelling}

\subsection{Computational domain}

To build the FE mesh, MRI (field strength of 1.5 Tesla) data consisting of $128 \times 128 \times 70$ voxels are used. The size of each voxel is $1.88 \times 1.88 \times 2 \mathrm{~mm}^{3}$. The axial slice of the geometry of the brain is extracted $\left(\mathrm{Comsol}^{\mathrm{TM}}\right.$ (http://www.Comsol.com)). In this slice, the two ventricles are visible and separated by a thin wall. The thickness of the slice is the same as that of the voxels, i.e. $2 \mathrm{~mm}$. Figure 6 shows the computation domain and the associated mesh. The mesh is made out of 63,104 prism elements with 64,398 nodes. The size of each mesh element is lower than the voxel size. Linear interpolation is used to have smooth variation of data over the domain. The mesh is refined in the region close to the ventricles.

The MRI and DTI acquisitions were done on a whole $3 \mathrm{D}$ brain of a given subject in the Geneva hospital. For the first study, a 3D slice was extracted as it helps us to distinguish the differences while avoiding the complexity of geometry. In addition, the slice was chosen in a way to include the regions of interest (Corona radiata and Corpus callosum).

\subsection{Constitutive equations}

Brain parenchyma is modelled as a porous medium fully saturated with CSF. The evolution of NPH is very slow and takes months or years. Therefore, steady state situation is assumed. Mechanical equilibrium in the two-phase medium requires that

$$
\operatorname{div}\left(\sigma_{t}\right)=\overrightarrow{0},
$$

where $\sigma_{t}$ is the total stress tensor. It is defined by the Terzaghi's principle that relates the stress over the solid phase 


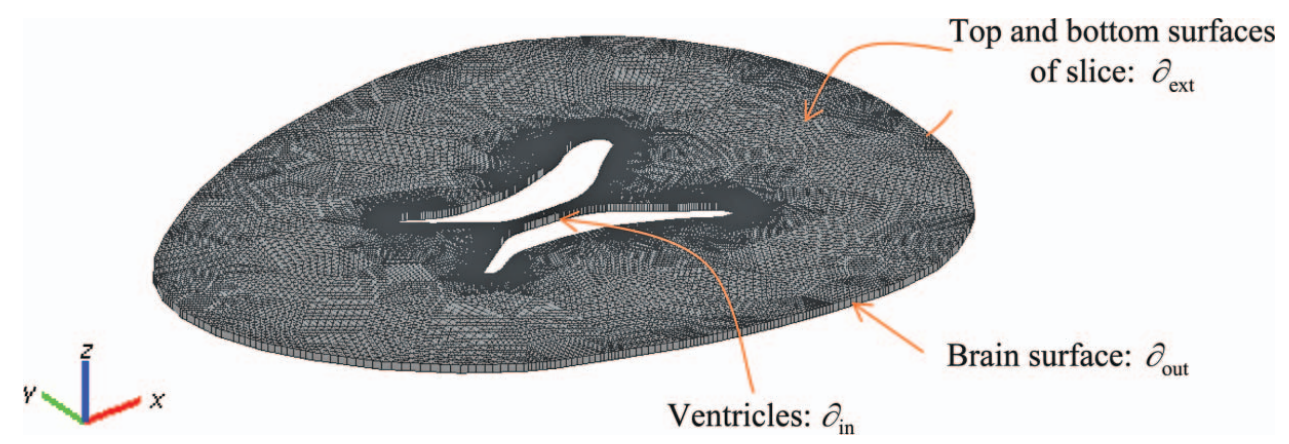

Figure 6. The 3D slice model with its boundaries.

$\sigma$ and the CSF pressure $p$ (Biot 1941; Wirth 2005) by

$$
\sigma_{t}=\boldsymbol{\sigma}+p \mathbf{I}_{d}
$$

where $\mathbf{I}_{d}$ is the identity tensor. The principle of effective stress, when applied to Hooke's law, yields (Biot 1941; Wirth 2005)

$$
\boldsymbol{\sigma}_{t}=\mathbf{C} \boldsymbol{\varepsilon}+p \mathbf{I}_{d}
$$

where $\mathbf{C}$ is the fourth order stiffness tensor that is defined by the Young's modulus, E, and Poisson's ratio, $v$, when it is assumed to be isotropic. $\boldsymbol{\varepsilon}$ is the Green-Lagrange's deformation tensor defined as

$$
\varepsilon=\frac{1}{2}\left(\nabla \vec{u}+\nabla \vec{u}^{T}+\nabla \vec{u} \times \nabla \vec{u}^{T}\right)
$$

where $\vec{u}$ is the displacement vector of the solid constituent. When assuming small deformation (SD), the third quadratic term of Equation (10) is ignored. CSF flow inside the medium is modelled with the help of the Darcy's law (Biot 1941):

$$
\vec{V}=-\frac{\mathbf{k}}{\mu}(\vec{\nabla} p),
$$

where $\vec{V}$ is the average CSF velocity $(\mathrm{m} / \mathrm{s}), \mathbf{k}$ the permeability tensor $\left(\mathrm{m}^{2}\right)$ and $\mu$ is the viscosity of CSF equal to that of water, i.e. 0.001 Pa.s. Taking the divergence of Equation (11) yields

$$
-\nabla \cdot(\mathbf{k} \vec{\nabla} p)=\operatorname{div}(\mu \vec{V})=0
$$

as it is assumed that there is no CSF sink or source inside the parenchyma. Finally, by putting Equation (8) into Equation (7) and using Equation (12), one gets two coupled differential equations that are solved numerically. The first one is for the mechanical equilibrium:

$$
\operatorname{div}(\sigma)+\vec{\nabla} p=\overrightarrow{0}
$$

The second equation is for the CSF mass conservation:

$$
\nabla \cdot(\mathbf{k} \vec{\nabla} p)=0
$$

Note that the permeability tensor $\mathbf{k}$ remains inside the divergence as it is highly space-dependent. The unknowns of the problem are $u_{x}, u_{y}$ and $u_{z}$, the three components of displacement of the solid constituent, and $p$ being the pressure of extracellular CSF, thus giving four degrees of freedom per node.

\subsection{Initial and boundary conditions}

Initial conditions are taken as zero for displacements and stresses at all mesh points:

$$
\vec{u}(t=0)=\overrightarrow{0} \text { and } \sigma(t=0)=0 .
$$

Initial CSF pressure is zero (i.e. $p(t=0)=0)$ and the extracellular CSF content is either $18 \%$ or non-uniform and defined by Equation (3). The calculation consists in finding the solution where the pressure within the ventricles is increased to $5 \mathrm{mmHg}$ (i.e. $666.61 \mathrm{~Pa}$ ) whereas it is kept to zero on the outer boundary. In other words, a pressure gradient of $5 \mathrm{mmHg}$ is applied between the ventricles and the SAS. The ventricles are assumed to be fully permeable and can deform freely. Thus,

$$
p=5 \mathrm{mmHg} \text { and } \sigma \vec{n}=-p \vec{n} \quad \text { on } \partial_{\text {in }}
$$

where $\vec{n}$ represents the normal vector on the ventricles' surface. Brain surface is assumed to be mechanically fixed:

$$
\vec{u}=\overrightarrow{0} \text { and } p=0 \text { on } \partial_{\text {out }} .
$$

The top and bottom surfaces of the slice are assumed to be impermeable and fixed for displacement along the $z$-direction, which leads to a quasi 2D situation throughout 
the slice (see Figure 6):

$$
u_{z}=0 \text { and } \frac{\partial p}{\partial \vec{n}}=0 \text { with } \vec{n}= \pm \vec{e}_{z} \quad \text { on } \partial_{\mathrm{ext}} .
$$

\section{Description of two simulation cases}

Two simulation cases are defined to study the influence of the non-uniform CSF content and anisotropy of the permeability on the CSF flow created by the imposed pressure gradient of $5 \mathrm{mmHg}(666.61 \mathrm{~Pa})$. The elastic properties of the brain are assumed to be isotropic and homogenous. A drained Poisson's ratio of $v=0.3$ is assumed for both cases based on the data used by other authors (Peña 2002; Momjian and Bichsel 2006). This Poisson's ratio accounts for some compressibility of the brain parenchyma. The shear modulus is chosen as an average value of $3.0 \mathrm{kPa}$ (Green et al. 2008). All boundary conditions are the same for both cases that are detailed hereafter.

\subsection{First case: homogeneous and isotropic}

In this case and similarly to most of the previous FE models of NPH, the permeability tensor reduces to one scalar coefficient $k$ taken as the average of the permeability coefficients over the slice in gray and white matters, i.e. $0.48 \times$ $10^{-14} \mathrm{~m}^{2}$ (cf. Equations (4-6)). In addition, initial CSF content is constant and uniform $f_{0}=18 \%$.

\subsection{Second case: anisotropic and inhomogeneous in permeability}

In this case, the inhomogeneity of the CSF content and anisotropy of the permeability tensor are introduced. The initial CSF content is space-dependent and defined by Equation (3). The permeability tensor is defined by the permeability coefficients given in Equations (4-6) in the local coordinate systems $(1,2,3)$ determined by DTI:

$$
\begin{aligned}
\mathbf{k}_{\text {gray }} & =\left[\begin{array}{ccc}
k_{\text {gray }} & 0 & 0 \\
0 & k_{\text {gray }} & 0 \\
0 & 0 & k_{\text {gray }}
\end{array}\right], \\
\mathbf{k}_{\text {white }} & =\left[\begin{array}{ccc}
k_{\text {perp }} & 0 & 0 \\
0 & k_{\text {perp }} & 0 \\
0 & 0 & k_{\text {para }}
\end{array}\right],
\end{aligned}
$$

as depicted in Figure 2, where direction (3) is the direction of highest diffusion.

\subsection{Solving strategy}

The two coupled differential equations are solved by the FE method. Direct solvers are more accurate but high memory consuming. The generalised minimal residual method (GMRES) iterative solver (Youcef and Martin 1986) present in Comsol is used with a geometric multigrid preconditioner. Quadratic interpolation functions are used for both displacement and pressure in each element. SD and LD theories are also tested. The time for the calculation of case 2 is about half a day on a double CPU personal computer with 2 GB of RAM. Several simulation runs are carried out on different mesh densities to ensure that the solution remains independent of the mesh grid.

\section{Results and discussion}

To ease the comparison between the two cases, the same scale is used. Figure 7 shows the ventricle dilation, the CSF pressure and streamlines under the imposed pressure difference of $5 \mathrm{mmHg}$ for both cases using SD. The ventricles dilate and compress the brain parenchyma towards the skull. Black lines represent the original ventricle shapes. The pressure field and CSF streamlines are distorted in case 2 compared to case 1, which exhibits the classic Laplacian solution of Equation (14) and has been retrieved in most studies of NPH. The pressure gradient close to the brain surface is lower in case 2 owing to the larger CSF content and the associated permeability coefficients. In the corpus callosum (cf. Figure 3), the direction of the highest diffusion is in plane and therefore CSF flow along that direction is facilitated as $k_{\text {para }}$, which is the highest permeability coefficient. On the other hand, the direction of the highest diffusion is perpendicular to the plane in the corona radiata. Thus the CSF flow is impeded owing to the lower value of $k_{\text {perp }}$.

The general pattern of displacements in both cases is very similar. This is not surprising, as the mean values of the permeability and the elasticity coefficients over the slice are the same. However, differences appear when plotting the displacement magnitude versus the arc-length, i.e. the distance from a base point on top of the ventricle horn counterclockwise, as shown in Figure 8 for the left ventricle. The increase in ventricle dilation from case 1 to case 2 is visible only in the region close to the corona radiata, i.e. for segment $\mathrm{AB}$, owing to the intricate relationship between the CSF content and the permeability coefficients. The increase is about $6 \%$ using $\mathrm{SD}$, while it increases up to $15 \%$ for $\mathrm{LD}$. Using LD theory together with non-uniform CSF content and anisotropic permeability, the displacement is increased by $53 \%$ compared to case 1 with SD. Ventricle dilation is the highest in case 2 with LD. Between the two ventricles, the left ventricle with a smaller initial volume dilates more. This correlates with the 'mechanical' explanation of NPH given by Hakim and Adams in 1965.

Figure 9(a) presents the initial fluid content calculated with Equation (3) and used in case 2 as an initial condition. Figures 9(b) and (c) show the final CSF distribution for cases 1 and 2, respectively. A specific colour-bar is used to 

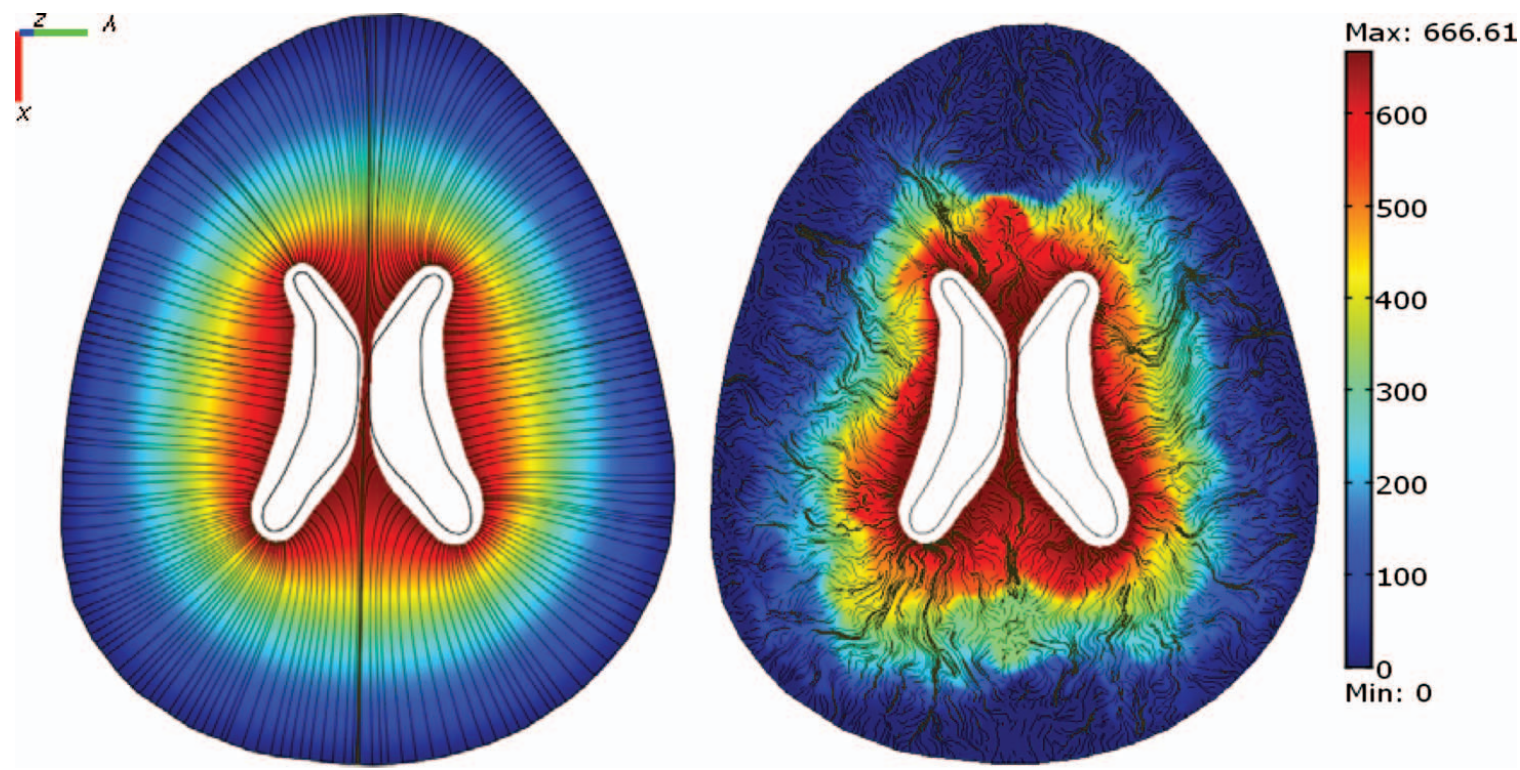

Figure 7. Ventricle dilation, pressure distribution (Pa) and CSF streamlines in cases 1 and 2 showing the large influence of non-uniform CSF content and anisotropic permeability.

include the values of CSF content from 0 to $100 \%$. This way there is no cut-off and the limit between the blue and yellow colours corresponds to the average CSF content of $18 \%$. Near the ventricle horns, the CSF content increases significantly for both cases (red region in Figures 9(b) and (c)). This concentration of CSF near the ventricle horns is generally accepted as the reason for periventricular lucency
(PVL) (Peña et al. 1999). On both sides of the ventricles the CSF content decreases, especially in case 2 (dark blue region in Figure 9(c)). This decrease may reduce the extracellular spaces in these regions, distort the blood vessels and lead to a collapse of the capillaries. This mechanism has been observed experimentally in hydrocephalic brains (Del Bigio 1993). In the remaining part of the brain parenchyma,

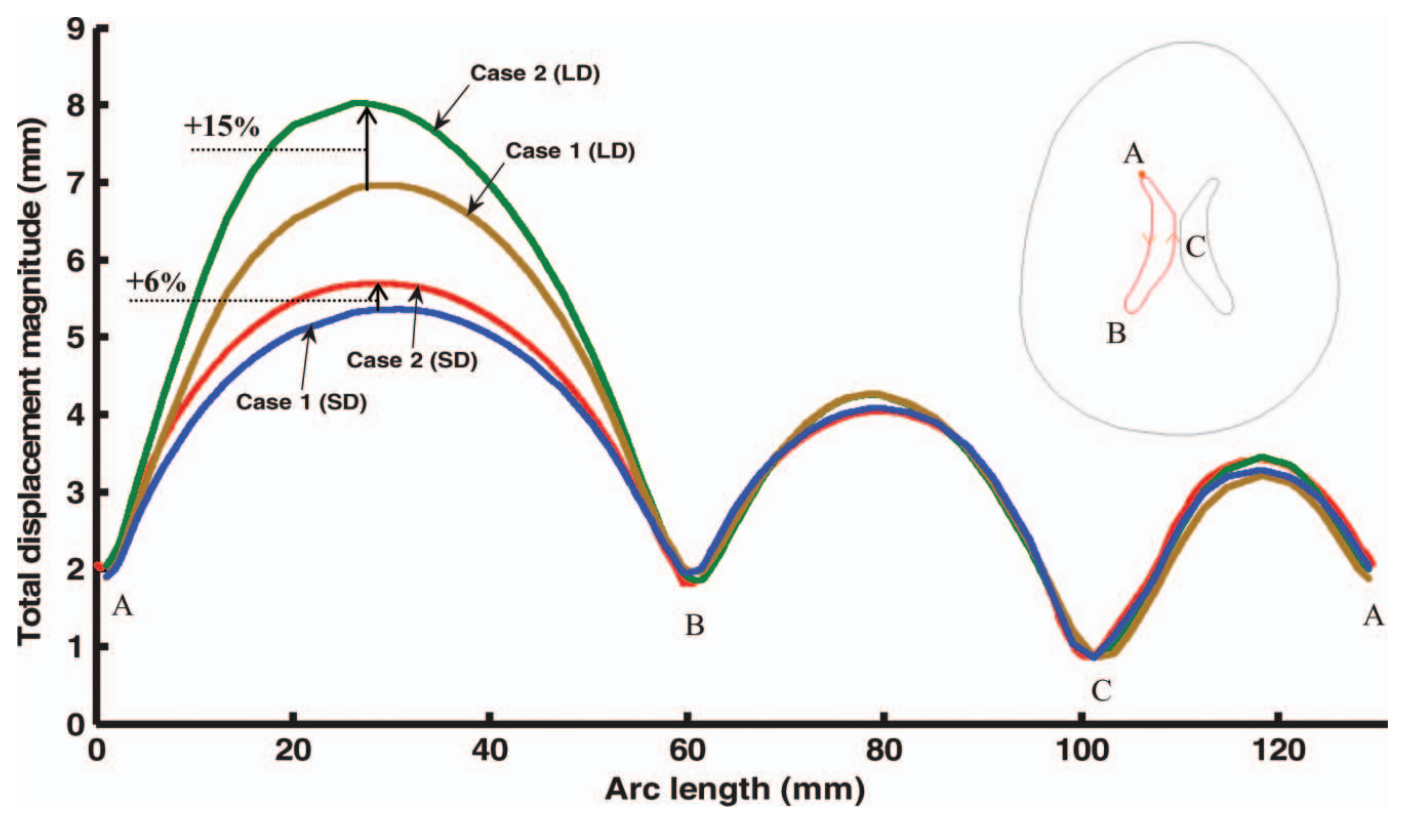

Figure 8. Displacement magnitude $(\mathrm{mm})$ in cases 1 and 2 including small deformation (SD) and large deformation (LD) analysis versus the arc-length $(\mathrm{mm})$ of the left ventricle. 


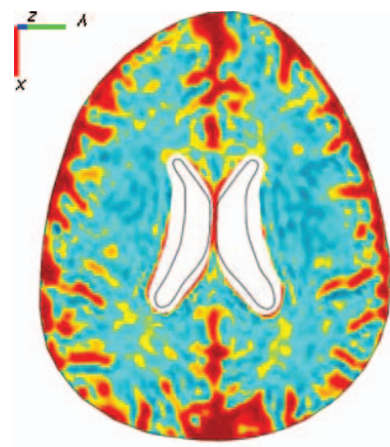

(a)

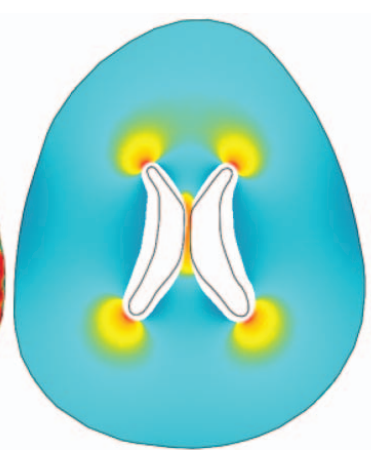

(b)

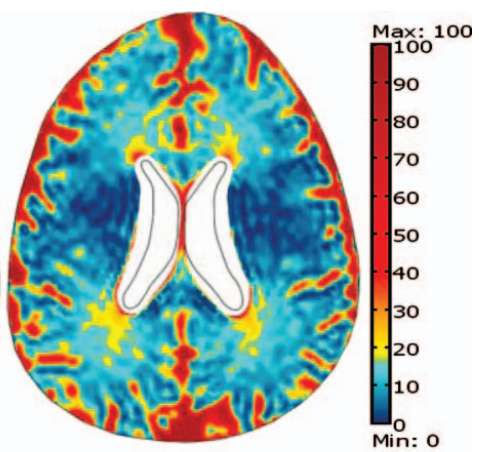

(c)

Figure 9. (a) Initial CSF content in case 2, (b) final CSF content in case 1 and (c) case 2.

the amount of CSF remains close to its initial value. However, it is important to note that the boundary conditions apply on the pressure itself (Dirichlet conditions, Equations (16) and (17)) and not on the CSF flux through the ventricle and brain surfaces that are assumed to be fully permeable. That is why the average CSF content increases for both cases under the 'mechanical' compression of the cerebral mantle.

Figure 10 shows the distribution of the CSF velocity for both cases using SD. A large influence of the CSF content and anisotropy in permeability is again observed in case 2 . In case 1 , the velocity is relatively uniform with minimum between the ventricles and maximum of $10 \mu \mathrm{m} / \mathrm{s}$ around the ventricle horns. This high velocity corresponds to the high CSF flow (or discharge) and is due to a geometrical effect on the Laplace's solution $\Delta p=0$. Case 2 yields a highly non-uniform velocity distribution with a large range of values going from almost zero between the two ventricles to $1000 \mu \mathrm{m} / \mathrm{s}$ in the CSF pathways that appear between the two hemispheres and close to the SAS. These pathways develop owing to the high local CSF content (cf. Equation (3)) and its influence on the permeability coefficients (cf. Equations (4) to (6)). This non-uniform pattern has been measured in NPH patients by Tullberg et al. (2001) using MRI (cf. Figure 10). With the help of the CINE-MRI technique, Linninger et al. (2009) reported such a non-uniform velocity distribution with a maximum value of around 2000 $\mu \mathrm{m} / \mathrm{s}$ in a hydrocephalic brain.

Figure 11 shows the $\sigma_{\mathrm{xx}}$ stress component of the solid stress tensor for both cases. The patterns look very similar owing to the fact that the elastic properties are identical. In both cases, the ventricle horns and the region between the two ventricles undergo tensile stresses along $x$, while the remaining part of the cerebral mantle is in compression. Part of the region corresponding to the corona radiata (cf. Figure (3)) with fibre directions perpendicular to the plane undergoes some tensile stresses. This tension can cause the fibre tracts to be detached from each other. This detaching mechanism has been described experimentally (Weller et al. 1971) and should be evidenced with the present model by introducing anisotropy in the elastic properties.

In the present study, a pressure gradient of $5 \mathrm{mmHg}$ is imposed between the ventricles and the SAS as previously done by Peña et al. (1999) and Momjian and Bichsel (2006, 2008). The existence of such a pressure gradient has never been demonstrated in the case of NPH. However, Levine
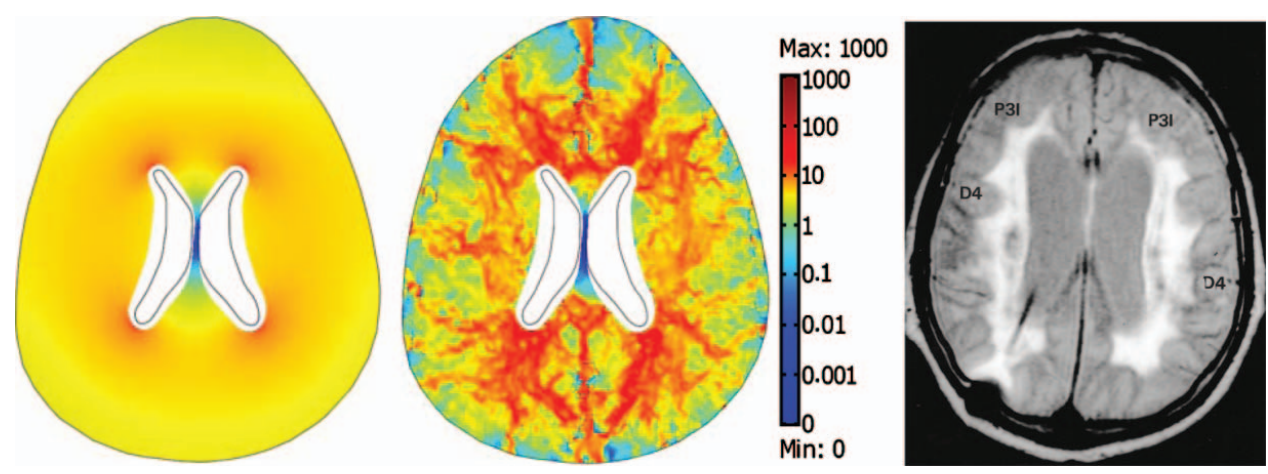

Figure 10. Fluid velocity distribution $(\mu \mathrm{m} / \mathrm{s})$ in cases 1 (left) and 2 (middle) with a realistic MRI scan of a NPH patient (right) (Tullberg et al. 2001)). 

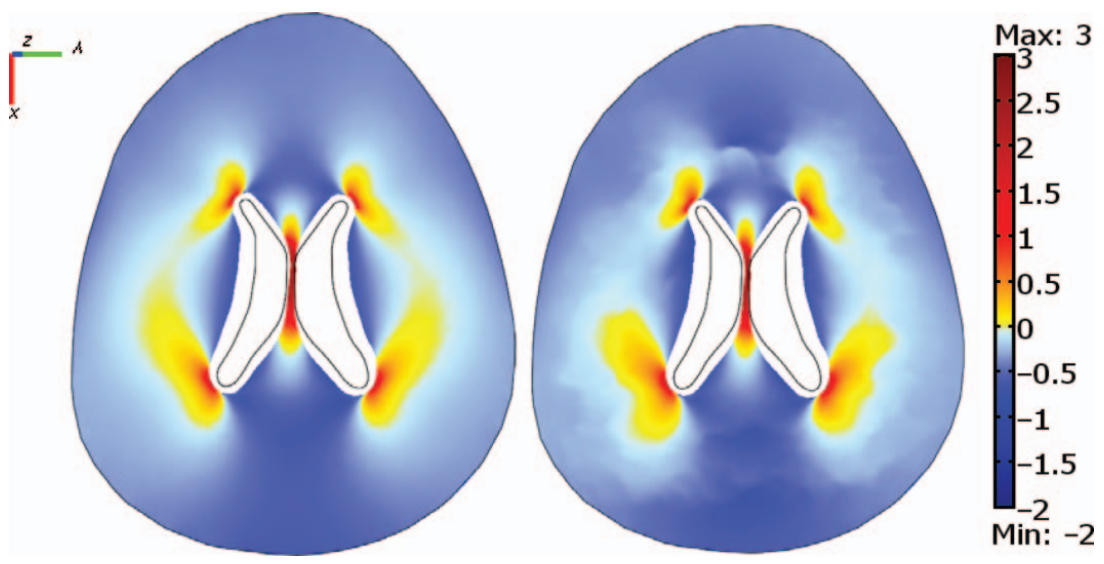

Figure 11. $\sigma_{x x}(\mathrm{kPa})$ plot in cases 1 and $2(\mathrm{SD})$.

(2008) recently raised the necessity of the existence of a mini-gradient lower than $1 \mathrm{mmHg}$ in the parenchyma. According to him, an increased transmantle pressure is needed to balance CSF production and CSF absorption. Nevertheless, the influence of anisotropy and inhomogeneity in permeability and LD effect should remain important in such a situation.

Due to the incompressible nature of the brain tissue, the undrained Poisson's ratio is very close to 0.5 . In the present study, a drained Poisson's ratio of 0.3 is used to consider the CSF discharge from parenchyma as both ventricle and brain membranes are assumed to be permeable. In reality, the CSF flow through these two membranes should be modelled by a Cauchy condition traducing some possible seepage. In that case, the CSF flow through the membrane is proportional to the difference in pressure on both sides. This aspect is not taken into account in the present model as data are currently missing.

\section{Conclusion}

The present study demonstrates the importance of both the inhomogeneity of the CSF content and the anisotropy of permeability in the ventricle dilation and in the CSF flow field. LD theory yields a larger increase in dilation and thus should be considered when the applied CSF pressure gradient is large. The CSF velocity field becomes much more inhomogeneous, as observed experimentally. With spacedependent CSF content and TI permeability, the proposed model becomes much more realistic. In a similar approach, TI elasticity tensor will be introduced in the future as well. Indeed, recent data based on magnetic resonance elastography (MRE) have shown that the white matter is stiffer along the fibre tracts than perpendicular to them.

In order to better understand the development of NPH the model still needs to be further improved, notably by using real 3D geometry. The brain and ventricle surfaces can be permeable to some extent. The existence of blood vessels plays an important role in the CSF circulation inside the parenchyma. The blood vessels and the arachnoids granulations behave as a sink absorbing CSF, whereas the choroid plexus produces CSF and behaves as a source. These effects of blood vessels and CSF production by the choroid plexus will be considered in the near future.

\section{Acknowledgements}

The financial support of the School of Engineering (STI) seed fund at EPFL is gratefully acknowledged. Our acknowledgements also go to Prof. Denis Bichsel from Geneva School of Engineering for his helpful collaboration.

\section{References}

Aboitiz F, Scheibel AB, Fisher RS, Zaidel E. 1992. Fiber composition of the human corpus-callosum. Brain Res. 598(12): $143-153$.

Basser PJ. 1992. Interstitial pressure, volume, and flow during infusion into brain tissue. Microvasc Res. 44(2):143-165.

Basser PJ, Mattiello J, LeBihan D. 1994. MR diffusion tensor spectroscopy and imaging. Biophys J. 66(1):259-267.

Basser PJ, Pierpaoli C. 1998. A simplified method to measure the diffusion tensor from seven MR images. Magn Reson Med. 39(6):928-934.

Bihan DL, Douek P, Argyropoulou M, Turner R, Patronas N, Fulham M. 1993. Diffusion and perfusion magnetic resonance imaging in brain tumors. Top Magn Reson Imaging. 5(2):2531.

Biot MA. 1941. General theory of three-dimensional consolidation. J Appl Phys. 12(2):155-164.

Carman PC. 1937. Fluid flow through granular beds. Trans Inst Chem Eng. 15:150-166.

Del Bigio MR. 1993. Neuropathological changes caused by hydrocephalus. Acta Neuropathol. 85(6):573-585.

Dutta-Roy T, Wittek A, Miller K. 2008. Biomechanical modelling of normal pressure hydrocephalus. J Biomech. 41(10):22632271.

Green MA, Bilston LE, Sinkus R. 2008. In vivo brain viscoelastic properties measured by magnetic resonance elastography. NMR Biomed. 21(7):755-764.

Gupta S, Soellinger M, Boesiger P, Poulikakos D, Kurtcuoglu V. 2009. Three-dimensional computational modeling of 
subject-specific cerebrospinal fluid flow in the subarachnoid space. J Biomech Eng. 131(2):021010-021011.

Hakim S. 1971. Biomechanics of hydrocephalus. Acta Neurol Latinoam. 1(Suppl):169-194.

Hakim S, Adams RD. 1965. The special clinical problem of symptomatic hydrocephalus with normal cerebrospinal fluid pressure: observations on cerebrospinal fluid hydrodynamics. J Neurol Sci. 2(4):307-327.

Kaczmarek M, Subramaniam R, Neff S. 1997. The hydromechanics of hydrocephalus: steady-state solutions for cylindrical geometry. Bull Math Biol. 59(2):295-323.

Larrat B, Chan QC, Yang XF, Li G, Yang ES, Fink M, Sinkus R. 2007. Anisotropic viscoelastic properties of the corpus callosum - application of high-resolution 3D MRElastography to an Alzheimer Mouse Model. Paper presented at: IEEE Ultrasonics International Conference, October 2007, NewYork.

Levine DN. 2008. Intracranial pressure and ventricular expansion in hydrocephalus: have we been asking the wrong question? J Neurol Sci. 269(1-2):1-11.

Linninger AA, Sweetman B, Penn R. 2009. Normal and hydrocephalic brain dynamics: the role of reduced cerebrospinal fluid reabsorption in ventricular enlargement. Ann Biomed Eng. 37(7):1434-1447.

Melhem ER, Mori S, Mukundan G, Kraut MA, Pomper MG, van Zijl PCM. 2002. Diffusion tensor MR Imaging of the brain and white matter tractography. Am J Roentgenol. 178(1):316.

Momjian S, Bichsel D. 2006. Elastic and poro-elastic models of ventricular dilatation in hydrocephalus. Proceedings of COMSOL Users Conference 2006, Grenoble, France.

Momjian S, Bichsel D. 2008. Nonlinear poroplastic model of ventricular dilation in hydrocephalus. J Neurosurg. 109(1):100107.

Nagashima T, Tamaki N, Matsumoto S, Horwitz B, Seguchi Y. 1987. Biomechanics of hydrocephalus: a new theoretical model. Neurosurgery. 21(6):898-904.
Peña A, Bolton MD, Whitehouse H, Pickard JD. 1999. Effects of brain ventricular shape on periventricular biomechanics: a finite-element analysis. Neurosurgery. 45(1):107.

Peña A, Harris NG, Bolton MD, Czosnyka M, Pickard JD. 2002. Communicating hydrocephalus: the biomechanics of progressive ventricular enlargement revisited. Acta Neurochir Suppl. 81:59-63.

Sivaloganathan S, Stastna M, Tenti G, Drake JM. 2005a. Biomechanics of the brain: a theoretical and numerical study of Biot's equations of consolidation theory with deformationdependent permeability. Int J Non-Linear Mech. 40(9):11491159 .

Sivaloganathan S, Stastna M, Tenti G, Drake JM. 2005b. A viscoelastic approach to the modelling of hydrocephalus. Appl Math Comput. 163(3):1097-1107.

Smillie A, Sobey I, Molnar Z. 2005. A hydroelastic model of hydrocephalus. J Fluid Mech. 539:417-443.

Tenti G, Sivaloganathan S, Drake JM. 1999. Brain biomechanics: steady state consolidation theory of hydrocephalus. CAMQ. 7(1):93-110.

Tullberg M, Jensen C, Ekholm S, Wikkelso C. 2001. Normal pressure hydrocephalus: vascular white matter changes on MR images must not exclude patients from shunt surgery. Am J Neuroradiol. 22(9):1665-1673.

Weller ROMDPD, Wisniewski HMDPD, Shulman KMD, Terry RDMD. 1971. Experimental hydrocephalus in young dogs: histological and ultrastructural study of the brain tissue damage. J Neuropathol Exp Neurol. 30(4):613-626.

Westhuizen JV, Du Plessis P. 1994. Quantification of unidirectional fiber bed permeability. J Compos Mater. 28(7):619-637.

Wirth B. 2005. A mathematical model for hydrocephalus [masters thesis]. [Oxford, UK]: University of Oxford.

Yaniv A, Tamar B-K, Yossi Y, Peter JB. 2008. Axcaliber: a method for measuring axon diameter distribution from diffusion MRI. Magn Reson Med. 59(6):1347-1354.

Youcef S, Martin HS. 1986. GMRES: a generalized minimal residual algorithm for solving nonsymmetric linear systems. SIAM J Sci Stat Comput. 7(3):856-869. 

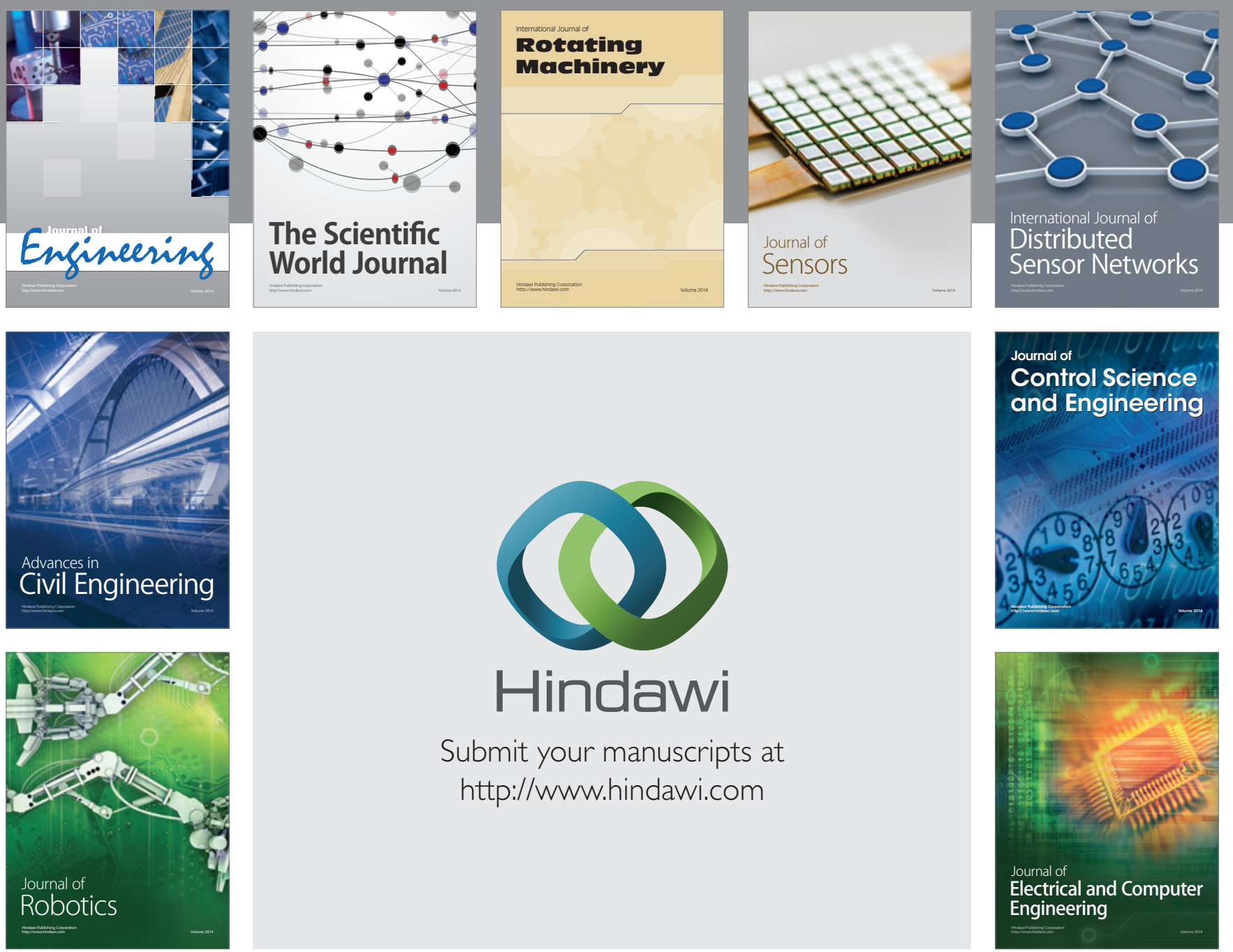

Submit your manuscripts at

http://www.hindawi.com
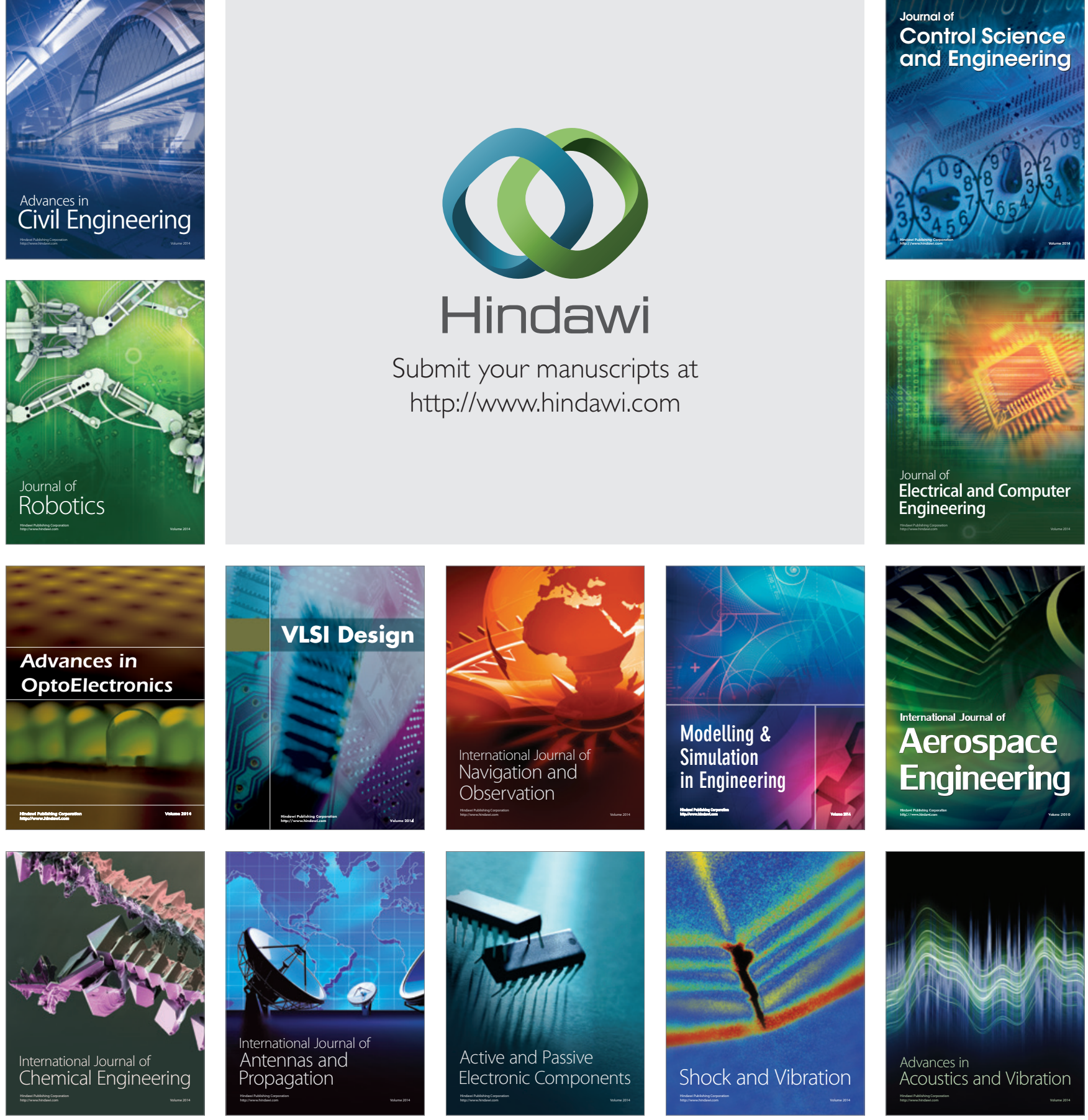